\title{
LOS LÍMITES DE LAS REFORMAS: VIOLENCIA CONTRA LAS MUJERES Y POLÍTICAS PÚBLICAS EN AMÉRICA LATINA
}

\section{THE LIMITS OF REFORM: VIOLENCE AGAINST WOMEN AND PUBLIC POLICY IN LATIN AMERICA}

\section{Montserrat Sagot*}

\author{
RESUMEN
}

En este artículo se analizan las políticas públicas para prevenir y combatir la violencia contra las mujeres que se han desarrollado durante las últimas dos décadas en América Latina. Se analiza también el papel de movimiento feminista y sus aportes en la reconceptualización de la violencia contra las mujeres, así como su influencia en la aprobación de normas legales y políticas sobre la problemática. Finalmente, se discute sobre la efectividad de las normas y políticas existentes y sobre las relaciones problemáticas entre el feminismo y el Estado en este terreno.

PALABRAS CLAVE: AMÉRICA LATINA * VIOLENCIA CONTRA LAS MUJERES * POLÍTICAS PÚBLICAS * GÉNERO * FEMINISMO * REFORMAS SOCIALES

\section{ABSTRACT}

This article analyses the public policy to prevent and confront violence against women, developed in Latin America during the last two decades. It also analyses the role of the feminist movement and its contributions to the re-conceptualization of violence against women, as well as its influence on the passing of legal norms and public policy on the subject. Finally, the article also discusses the effectiveness of those legal norms and policy and the problematic relations between the feminist movement and the State in this area.

KEY WORDS: AMÉRICA LATINA * VIOLENCE AGAINST WOMEN * PUBLIC POLICY * GENDER * FEMINISM * SOCIAL REFORM

Escuela de Sociología y Centro de Investigación en Estudios de la Mujer, ambos de la Universidad de Costa Rica.

msagot@cu.ucr.ac.cr 
Aunque las mujeres siempre se han resistido a la violencia sexista en forma individual, e incluso se han atrevido a denunciarlo públicamente, (en Costa Rica hay ejemplos de denuncias por violencia doméstica, incesto y violación desde los siglos XVIII y XIX) ${ }^{1}$ antes de 1970 eran muy pocos los que hablaban de mujeres maltratadas, abusadas o víctimas de incesto. La mayoría de las personas, tanto de la Sociedad Civil, como de la academia y de los gobiernos, no pensaba que estas situaciones afectaran a un número significativo de mujeres como para considerarlo un problema grave o digno de atención. Sin embargo, en un proceso que originalmente fue de comunicación personal de mujer a mujer, se empieza a hablar del asunto, a organizar los primeros grupos $y$ a hacer las primeras investigaciones.

Gracias a estas nuevas iniciativas la violencia contra las mujeres ha sido reconocida durante las últimas décadas como un problema social de grandes dimensiones. A pesar de los avances en la visibilización del problema, en la provisión de servicios para las afectadas y en la aprobación de tratados internacionales, legislación nacional y políticas públicas, este tipo de violencia sigue causando más muertes $y$ daños en las mujeres de 15 a 44 años que la malaria, el sida o la guerra (Carcedo y Zamora, 1999; World Health Organization, 2005).

Diversos estudios han mostrado que la violencia contra las mujeres es un problema social de gran magnitud, producto de una organización social estructurada sobre la base de la desigualdad de género, que afecta sistemáticamente a millones de mujeres en todo el mundo (Heise, Pitanguy y Germain, 1994; Ellsberg, et ál., 1996; Carcedo y Zamora, 1999; Kennedy, 1999, Sagot y Carcedo, 2000; Johnson, et ál., 2008). Una forma endémica de este tipo de violencia es el abuso de las mujeres por parte de su pareja. Según investigaciones realizadas

Datos revelados en las investigaciones de la historiadora Eugenia Rodríguez (2006 y 2000), tales como Divorcio y violencia de pareja en Costa Rica (1800-1950) e Hijas, novias y esposas. Familia, matrimonio y violencia doméstica en el Valle Central de Costa Rica (1750-1850). en América Latina, entre un cuarto y más de la mitad de las mujeres reportan haber sido maltratadas en algún momento por sus parejas (Heise, Pitanguy y Germain, 1994; Johns Hopkins University-CHANGE, 1999; United Nations, 2000; Güezmes, Palomino y Ramos, 2002; Sagot, 2004). En Costa Rica, los resultados de la Encuesta Nacional de Violencia Contra las Mujeres, mostraron que el 58\% de las mujeres había experimentado, al menos, un incidente de violencia física o sexual desde los 16 años y que, en la mayoría de las ocasiones, esa violencia había sido ejercida por un hombre cercano (Sagot, 2004).

La violencia contra las mujeres es un componente estructural del sistema de opresión de género. El uso de la violencia es no sólo uno de los medios más efectivos para controlar a las mujeres, sino también una de las expresiones más brutales y explícitas de la dominación y la subordinación. La posición de mujeres y hombres se organiza como una jerarquía en la que los hombres tienen control sobre los principales recursos de la sociedad y sobre las mujeres. Existen numerosos soportes ideológicos, morales, políticos, económicos y legales para el ejercicio de la autoridad de los varones sobre las mujeres. Aunque estos soportes varían histórica y culturalmente, el uso de la violencia constituye una de las formas más predominantes $y$ generalizadas que ayudan al ejercicio de esa autoridad.

Como lo afirman Rebecca y Russell Dobash (1979), si bien el derecho legal de los hombres a ejercer violencia contra las mujeres ya no es explícitamente reconocido en la mayoría de las sociedades occidentales, el legado de leyes antiguas y de prácticas sociales abiertamente aprobadas continúan generando las condiciones que permiten la existencia generalizada de este tipo de violencia. Aunque las leyes ya no brindan un apoyo explícito a esta práctica, la inacción, la indiferencia, las políticas y procedimientos contradictorios de las instituciones sociales continúan reflejando el ideal de la posición subordinada de las mujeres y el derecho de los hombres a dominar y controlar, hasta haciendo uso de la violencia. Asimismo, la histórica inacción estatal en este terreno refleja la concepción de que ciertos aspectos de la vida 
social, en particular los que se configuran dentro del hogar o en el ámbito denominado "privado", deben estar fuera del control del Estado.

Lo anterior es el resultado de ancestrales concepciones que se remontan a Aristóteles, cuya teoría de la justicia relegaba a las mujeres a la esfera del hogar, un lugar ocupado por personas que no eran iguales a los hombres libres, aquellos que podían participar activamente en la justicia política. De hecho toda la teoría política occidental y consecuentemente la configuración de las instituciones sociales ha estado influenciada por estas concepciones que consideran a la mitad de la humanidad - 0 la cuasi-humanidad, es decir a las mujerescomo encargadas de áreas de la vida definidas como fuera del ámbito de la justicia (Moller Okin, 1989).

Por otra parte, en las sociedades occidentales las ideas de paz y seguridad están fuertemente asociadas con la institución de la familia, tanto que algunos pensadores conservadores, como Talcott Parsons (1976), le han llamado "el paraíso en un mundo sin alma". Como consecuencia, ha sido y es todavía difícil aceptar el hecho de que la mayoría de las violaciones a los derechos humanos de las mujeres tienen lugar dentro del hogar o en el contexto de las relaciones cercanas. Pero la verdad es que para las mujeres, la familia es el grupo social más violento y el hogar el lugar más peligroso. De hecho, la Encuesta Nacional de Violencia Contra las Mujeres de Costa Rica demostró que los hombres con quienes las mujeres tienen relaciones familiares, de afinidad o convivencia cometen más del $65 \%$ de los actos de violencia. Asimismo, se comprobó la peligrosidad del ámbito "privado" ya que es en el hogar donde ocurren más del $80 \%$ de los incidentes de violencia, principalmente los perpetrados por compañeros íntimos, así como la mayoría de los asesinatos de mujeres o femicidios (Sagot, 2004; Carcedo y Sagot, 2002; Pola, 2002).

Desde esa perspectiva, la violencia basada en la inequidad de género es un elemento central que ayuda a comprender la condición social de las mujeres. La presencia o amenaza real de violencia cotidiana ilustran cómo la opresión y la desigualdad colocan a las mujeres en una posición de gran vulnerabilidad. La violencia contra las mujeres es de hecho la piedra angular de la dominación de género. Como lo plantea Lori Heise: "esta violencia no es casual, el factor de riesgo es ser mujer. Las víctimas son elegidas por su género. El mensaje es dominación: confórmate con tu lugar" (Heise, citada por Bunch, 1991: 8).

Ha sido el movimiento de mujeres y en particular el movimiento feminista, el que, desde la década de los años 70, inició la visibilización de esta violencia como un problema social y político. Existe una voluminosa producción teórica y empírica que demuestra que la puesta en la agenda pública de esta problemática y la consecuente aprobación de legislación y políticas públicas para enfrentarla es el resultado directo de las acciones del movimiento feminista (Pleck, 1987; Gordon, 1988; Sullivan, 1994; Sagot y Carcedo, 2000; Carcedo y Molina, 2003; Lazarus-Black, 2003; Weldon, 2004; MacDowell Santos, 2004). Se inicia así un proceso para tratar de romper con el mito de que esta forma de violencia es un asunto "privado" y hasta normal dentro de la dinámica familiar y humana, por lo que debe mantenerse fuera de los alcances de la justicia y de la intervención del Estado. Por medio de argumentos, presentación de testimonios y resultados de investigación, manifestaciones $y$ otras intervenciones expresivas, el movimiento feminista logró sacar el problema a la luz, con lo que contribuyó, además, a mostrar que la dicotomía entre lo público y lo privado, $y$ entre lo personal $y$ lo político es artificial, y consecuentemente, abrió el camino para que se plantearan demandas concretas de intervención estatal.

\section{HACIENDO VISIBLE LO IGNORADO: LOS APORTES DEL MOVIMIENTO FEMINISTA}

La violencia contra las mujeres, como un problema de gran magnitud y graves consecuencias, comenzó a ser puesto en la palestra pública y a ser denunciado por el movimiento feminista en América Latina en la década de los años 80. Durante toda la década, aunque con diferentes ritmos, las organizaciones feministas de los distintos países de la región realizaron campañas de denuncia y sensibilización, 
elaboraron propuestas de legislación y políticas públicas e iniciaron la apertura de programas de atención ${ }^{2}$.

En los primeros años de la década de los 90 se crean, en casi todos los países, redes nacionales contra la violencia hacia las mujeres, conformadas principalmente por organizaciones feministas, organismos no gubernamentales $y$ mujeres independientes vinculadas a esta problemática. En aquellos países en que no se han constituido redes, se han generado articulaciones específicas para realizar acciones y conmemorar el 25 de noviembre, Día Internacional de la No Violencia contra las Mujeres ${ }^{3}$.

Estos hitos latinoamericanos tienen como antecedentes internacionales a la segunda Conferencia Mundial de Naciones Unidas sobre la Mujer (1980), donde se adopta la resolución titulada "La mujer maltratada y la violencia en la familia” y el proceso que llevó a la aprobación de la Convención para la Eliminación de Todas las Formas de Discriminación contra la Mujer (CEDAW), principal instrumento jurídico a escala internacional para promover la igualdad de las mujeres.

En el ámbito gubernamental, la acción para erradicar la violencia contra las mujeres era todavía incipiente en este período. Parte importante de los países de la región se encontraba bajo dictaduras militares o situaciones de conflictos armados o estaban apenas saliendo de ellas. Por esto, las oportunidades que ofrecía el contexto político para incorporar este tema en las agendas de los gobiernos eran difíciles $y$, en algunos casos, claramente adversas. Las

2 El primer programa de esta naturaleza fue la Casa Protegida "Julia Burgos" abierta en Puerto Rico en 1979.

3 La fecha fue elegida en conmemoración del brutal asesinato en 1960 de las tres hermanas Mirabal (las Mariposas), activistas políticas de la República Dominicana, por orden del dictador Rafael L. Trujillo (1930-1961). Siguiendo la conmemoración instaurada por el movimiento feminista, en diciembre de 1999, la 54ª sesión de la Asamblea General de las Naciones Unidas adoptó la Resolución 54/134 en la que declaró el 25 de noviembre Día Internacional de la Eliminación de la Violencia contra la Mujer. primeras legislaciones en la materia fueron promulgadas en Puerto Rico en 1988 y 1989 (Leyes sobre el hostigamiento sexual en el trabajo y sobre violencia doméstica en la pareja). Asimismo, en Brasil, en 1988 se incluyó en la Constitución Federal una provisión con la cual el Estado se comprometía a crear mecanismos para cohibir la violencia en el ámbito de las relaciones familiares. En este mismo país, desde 1985, se crearon las Delegaciones de la Mujer, al igual que ocurrió en Perú y Argentina en 1988 (Isis Internacional, 2002; Debert, 2006).

Un elemento fundamental utilizado por el movimiento feminista en este proceso fue la ubicación de la violencia contra las mujeres como un problema de poder, es decir producto de un sistema estructural de opresión de género. Si bien la relación entre violencia y poder ha sido planteada en diversas ocasiones por las Ciencias Sociales, esta fue, quizá, la ocasión en que resultó más difícil hacerlo ya que existían numerosos elementos que primero ocultaban y después justificaban los comportamientos agresivos de los hombres hacia las mujeres (causas innatas o biológicas, drogas, alcohol, pobreza, provocación de las mujeres, masoquismo de las mujeres, etc.). Un hilo conductor de este proceso fue el relacionar la violencia contra las mujeres con la subordinación de género que existe en todas las sociedades. En ese sentido, desde el movimiento feminista se afirma que esta violencia no es casual ni coyuntural, y se realiza un análisis de las diferentes instituciones $y$ prácticas sociales como reflejo de esta manifestación por excelencia de la dominación masculina. Como lo expresa Ana Carcedo:

Hemos hablado de las leyes hechas por los hombres para mantener su dominación de género; de la policía como guardiana de ese orden patriarcal; de las instituciones estatales insensibles al dolor $y$ las necesidades de las mujeres maltratadas; de la educación que acuña hombres agresores y mujeres desempoderadas; de las iglesias con sus discursos de perdón; de la moral judeocristiana de culpa $y$ renuncia a la felicidad en este mundo... Y también de la estructura de propiedad y crédito que coloca los recursos 
económicos en manos masculinas; de la estructura política formal que excluye a las mujeres... y de las costumbres y tradiciones que son prácticas estereotipadas, ritos de mantenimiento del orden establecido entre géneros (Carcedo y Molina, 2003: 7).

El movimiento feminista también ha contribuido a comprender que si bien la socialización de género fomenta y propicia la agresión contra las mujeres, esta no es su causa de fondo ya este proceso no ocurre en el vacío. El trasfondo del proceso de socialización genérica es una estructura social de inequidad y opresión. El objetivo central de la socialización es obligar a las personas a adaptarse a las normas de su sociedad y, esta sociedad está estructurada con base en la desigualdad y la opresión de género. En ese sentido, el proceso de socialización de género reproduce y justifica en cada individuo esas jerarquías, principalmente la jerarquía entre los sexos, y los patrones de dominación y opresión institucionalizada existentes, ya que la transmisión de la ideología de la opresión es el elemento central de la socialización (Sagot, 1994).

Por otra parte, el movimiento feminista latinoamericano también ha planteado este problema como una negación de derechos ciudadanos, de seguridad pública y de exclusión social. En ese sentido, la violencia contra las mujeres ha sido concebida como un asunto de justicia. Es decir, no como un problema individual, privado, familiar o relacional, sino como un problema público, estrechamente vinculado con la ciudadanía, esto es, con la distribución social de los derechos y deberes, de las cargas y los beneficios, donde las mujeres, en particular las abusadas, se encuentran en una situación de negación de sus derechos y beneficios.

Las feministas han demandado de las instituciones sociales y políticas de sus países una concepción amplia de justicia social que permita la construcción de una sociedad que contenga y sustente las condiciones sociales necesarias para que todos sus miembros, según su condición particular, desarrollen y ejerzan sus capacidades, expresen sus experiencias y participen en la determinación de sus condiciones de vida. No se hace referencia a un concepto de justicia universalizante, sino a uno que tome en cuenta las particularidades $y$ las diferencias que las jerarquías sociales y sexuales producen en los individuos y que determinan su acceso diferenciado a los recursos materiales y simbólicos de la sociedad. En ese sentido, se ha demandado una "generización" del Estado (Lazarus-Black, 2003); es decir, un Estado que explícitamente reconozca que algunos ciudadanos son hombres $y$ otras son mujeres, $y$ un cambio en las relaciones de poder para garantizar a las mujeres control sobre los recursos materiales y simbólicos de la sociedad y sobre sus propios cuerpos, lo que significa control sobre su propia vida.

El empoderamiento y la garantía de una ciudadanía activa para las mujeres y todos los grupos excluidos son procesos íntimamente interconectados. El empoderamiento se relaciona, antes que nada, con el cambio en las relaciones de poder a favor de aquellos que tienen poco control sobre sus condiciones de vida. La ciudadanía, a su vez, puede ser definida como el derecho creciente a tener derechos universales $y$ particulares. El proceso de empoderamiento implicaría entonces el derecho a tener control sobre los recursos (económicos, físicos, intelectuales, etc.) y sobre el propio ser, y el derecho a tener control y participación en los procesos ideológicos y políticos (producción del conocimiento, construcción de valores, toma de decisiones, etc.).

Desde la anterior perspectiva, según el pensamiento feminista, la violencia contra las mujeres implica una negación de derechos ciudadanos para las mujeres, lo que las coloca en una situación de desempoderamiento y exclusión social. En ese sentido, la erradicación de la violencia implicaría un cambio en las relaciones de poder que inciden tanto en lo personal como en lo colectivo. Contrario entonces al discurso liberal, el feminismo argumenta que la violencia contra las mujeres ilustra claramente cómo la construcción de ciudadanía está estrechamente vinculada con el derecho a tener control sobre la vida personal y el propio cuerpo, y no solo con la garantía de derechos civiles, económicos o sociales. No hay ciudadanía cuando no se tiene poder individual, pero tampoco hay ciudadanía cuando como grupo social no se participa 
en la toma de decisiones y la definición de los destinos de la sociedad.

Desde la reflexión feminista también se ha establecido la relación entre los distintos tipos de violencia, desde la psicológica o emocional hasta la política. Se ha planteado, además, la similitud en sus causas, es decir, un sistema social estructurado sobre la base de las jerarquías, la desigualdad y la opresión. Se ha demostrado cómo, hasta en las guerras, las mujeres siempre llevan la peor parte, aunque no participen directamente en las batallas. En los conflictos bélicos, las mujeres, como civiles, tienen más posibilidades de morir que los soldados. Con las nuevas tecnologías de guerra, cada vez más letales y eficientes, la población civil corre mayor riesgo (Lorentzen y Turpin, 1998).

De hecho, las muertes de civiles en las guerras han ido aumentado, $y$ para los años 90, la población civil ya aportaba el 90\% de las muertes en estos conflictos. Las mujeres, las niñas y los niños constituyen la vasta mayoría de las víctimas (United Nations, 2000). Asimismo, la violación contra las mujeres es un arma usada sistemáticamente por los perpetradores de las masacres. Igualmente, las mujeres son violadas en los campamentos de refugiados o en los procesos de desplazamiento y son sometidas a la explotación sexual y otras formas de abuso en los períodos bélicos.

Dadas las situaciones de conflicto social y político de gran envergadura y de violación sistemática a los Derechos Humanos que se vivieron en la región, algunas organizaciones del movimiento feminista de Centroamérica desarrollaron su planteamiento de que los procesos de paz y democratización necesitan garantizar una vida libre de violencia y abuso de poder a toda la población, sin importar el espacio donde se ejerza esa violencia o quienes sean los perpetradores. Como lo han expresado algunas feministas, fundadoras del movimiento por la no violencia contra las mujeres, era importante posicionar esa concepción:

Máxime cuando en la vida cotidiana todas las formas de violencia están profundamente entrelazadas, siendo en ocasiones los mismos agresores, en el ejercicio de los diversos poderes que administran, quienes practican esas diversas formas de violencia (Carcedo y Verbaken, 1992: 12).

De esta manera, frente a las propuestas y firmas de acuerdos de paz en Centroamérica las organizaciones feministas plantearon que no podía haber paz si esta no comenzaba en casa. Un lema similar ya había sido usado por las chilenas cuando, en su lucha contra la dictadura de Pinochet, demandaron "democracia en la calle y en la casa".

En la década de los años 90 el movimiento feminista consiguió algunas de sus más relevantes conquistas, ya que logró colocar la violencia contra las mujeres en la agenda mundial y trasladó parte de sus concepciones a declaraciones internacionales. Después de varias resoluciones de las Naciones Unidas y de la Organización de Estados Americanos, en la Conferencia Mundial de Naciones Unidas sobre Derechos Humanos (Viena, 1993) se logró cristalizar un consenso político de que las diversas formas de violencia contra las mujeres, tanto las que ocurrían en el marco de conflictos armados y otros escenarios públicos, como las que ocurrían en la vida privada, debían ser conceptualizadas como violaciones flagrantes a los derechos humanos (Sullivan, 1994).

En nuestro continente, como resultado también de la acción feminista, en 1993 la Organización Panamericana de la Salud declaró la violencia contra las mujeres como un problema de salud pública y en 1994 la Organización de Estados Americanos aprobó la Convención Interamericana para Prevenir, Sancionar y Erradicar la Violencia contra las Mujeres (Convención de Belém do Pará), la que tiene un carácter vinculante para los Estados parte. Asimismo, en la Conferencia Mundial de Naciones Unidas sobre la Mujer (Beijing, 1995), a raíz de la propuesta de las latinoamericanas, se incluyó la violencia contra las mujeres como uno de los doce puntos de la Plataforma de Acción.

Estos instrumentos $y$ declaraciones internacionales le han sido de gran utilidad al movimiento feminista para demandar acciones particulares en cada país, argumentando la responsabilidad que le compete a los Estados, tanto por acción como por omisión (Isis Internacional, 2002). Como consecuencia, 
en la década de los años 90 todos los países del continente, a excepción de Estados Unidos, ratificaron la Convención de Belém do Pará y aprobaron legislación y políticas públicas relativas a algunas formas de violencia, en particular la intrafamiliar. También se han desarrollado planes nacionales para abordar la violencia, proyectos de investigación, programas académicos y programas de capacitación para personal de las instituciones públicas y privadas. Asimismo, se han multiplicado las propuestas de atención, así como la creación de comisarías de la mujer y albergues para mujeres maltratadas, en algunos países. Se ha producido también un avance significativo en términos de la conceptualización y aclaración de lo que se entiende por violencia contra las mujeres, así como en la tipificación de los actos violentos, dejando cada vez menos margen a la interpretación subjetiva (Isis Internacional, 2002).

Como parte de los nuevos instrumentos desarrollados desde el feminismo, se han planteado, además, algunos parámetros para valorar las leyes y políticas en materia de violencia contra las mujeres. Algunos de estos parámetros son:

$\diamond$ La violencia debe ser abordada $y$ conceptualizada como una manifestación de las relaciones desiguales de poder entre mujeres y hombres.

$\diamond$ Las leyes, políticas y programas deben servir para empoderar a las mujeres individual $y$ colectivamente, $y$ nunca para revictimizarlas.

$\diamond$ Las normas deben ser efectivas para enfrentar en la práctica la naturaleza y magnitud del problema, así como sus diversas manifestaciones.

$\diamond$ En conjunto, las leyes y políticas deben contemplar todas las dimensiones señaladas por la Convención de Belém do Pará: la prevención, la protección, la sanción y la reparación integral del daño (CEFEMINA, 2003).

\section{PRINCIPALES LOGROS DEL MOVIMIENTO FEMINISTA}

En los últimos 20 años se ha experimentado un avance importante en todos los países de la región. En términos de la legislación sobre violencia contra las mujeres, a fines de los años ochenta sólo un país, Puerto Rico, había legislado sobre esta materia. Para la mitad de la presente década, la mayor parte de los países ha abordado la problemática de alguna manera, por medio de la aprobación de normas específicas o modificando legislaciones anteriores. De hecho, varios países han adoptado también disposiciones constitucionales relacionadas con el derecho de las mujeres a una vida libre de violencia, lo que reviste especial importancia si se considera que este es el instrumento jurídico que enmarca el resto de la legislación nacional.

En relación con las normas específicas sobre violencia contra las mujeres, según se planteó, estas revisten básicamente dos modalidades: leyes especiales sobre la materia y modificación de los códigos penales. Las leyes aprobadas son de dos tipos: las que establecen medidas de protección y las que sancionan; es decir, que establecen procedimientos que pueden terminar en la sanción del agresor. Por otra parte, en varios países se han modificado los códigos penales para que expresamente se considere y sancione las diversas manifestaciones de la violencia contra las mujeres.

Muchos de los países de la región han realizado también modificaciones tendientes a normar, tipificar y sancionar la violencia sexual. De acuerdo con Tamayo (2000), las modificaciones en este sentido han logrado que los delitos sexuales dejen de ser tipificados como delitos contra el honor $y$ las buenas costumbres, $y$ pasen a ser considerados como "delitos contra la integridad o la libertad sexual”. Sin embargo, en algunos países, se conservan todavía normas legales que mantienen términos referidos a la honestidad de la mujer o que establecen disposiciones para eximir de la pena al agresor si contrae matrimonio con la víctima (Brasil, Nicaragua, Panamá y Uruguay).

Otra manifestación de la violencia sexual que ha sido incorporada en la normativa de algunos países es el hostigamiento o acoso sexual. Algunas de las modificaciones señaladas arriba incluyen $y$ tipifican esta figura, tal es el caso de República Dominicana, Honduras, Ecuador, Paraguay y El Salvador. En cuanto a normativas específicas, Argentina sancionó 
en 1993 un Decreto sobre acoso sexual en la Administración Pública y Costa Rica aprobó la Ley contra el hostigamiento sexual en el empleo y la docencia (1995). A pesar de estos importantes avances, es necesario destacar que esta figura no está tipificada en muchos de los países de la región, lo que constituye un vacío legal importante.

Así como existe un avance importante en términos normativos, también lo hay en la elaboración e implementación de políticas públicas dirigidas a abordar la problemática. La existencia de estas políticas se debe, en parte importante, a la instalación de mecanismos nacionales para la protección y ampliación de los derechos de las mujeres (ministerios, oficinas o institutos de la mujer), desde los que se ha impulsado la elaboración de políticas públicas, tanto nacionales como sectoriales. En ese sentido, la mayor parte de los países cuenta con alguna política, plan o programa nacional para hacer frente a esta manifestación de la violencia. Además de las políticas nacionales señaladas, en los distintos países de la región se han generado también algunas políticas sectoriales, principalmente en el sector salud.

Estas políticas usualmente se ejecutan por medio de acciones conjuntas entre los organismos coordinadores $y$ las comisiones nacionales o sectoriales creadas para tal efecto. En estas comisiones participan instituciones estatales, académicas $y$, en muchos casos, organizaciones de la sociedad civil, lo que ha resultado en un interesante ejercicio de articulación de esfuerzos y generación de estrategias de cogestión entre el Estado y la sociedad civil. Esta articulación de esfuerzos ha generado también servicios de apoyo específicos para las personas afectadas por la violencia, tales como las Comisarías de la Mujer y centros de atención psicológica y legal. En unos pocos países se han creado también albergues y casas refugio para mujeres maltratadas (Bolivia, Brasil, Ecuador, Panamá, Costa Rica). En el marco de estas políticas, además de los servicios señalados, se han generado campañas de prevención y sensibilización a la opinión pública, así como importantes acciones de capacitación a funcionarios y funcionarias públicas.
Por su parte, desde las organizaciones feministas $y$ del movimiento de mujeres en general se han llevado a cabo diversas campañas de sensibilización frente a la problemática. Estas se han realizado principalmente alrededor del día 25 de noviembre, utilizando distintos lemas y abordando aquellos temas que surgen como relevantes para los países en determinados momentos. En el caso de Costa Rica, durante varios años se utilizó esta fecha para denunciar los asesinatos de mujeres y para demandar a la Asamblea Legislativa la aprobación de la Ley de Penalización de la Violencia contra las Mujeres.

Estas campañas tienen diversos objetivos, como visibilizar y sensibilizar frente a la violencia contra las mujeres, hacer interlocución con el Estado, fiscalizar el cumplimiento de los compromisos nacionales e internacionales y denunciar ante la comunidad local, nacional e internacional las violaciones a los derechos humanos de las mujeres. De acuerdo con la evaluación de las integrantes de la Red Latinoamericana y del Caribe contra la Violencia Doméstica y Sexual, los mayores éxitos de las campañas han sido la sensibilización y visibilización de la problemática ${ }^{4}$. Así, para el feminismo y el movimiento social de mujeres un importante avance en la última década fue la articulación de esfuerzos, tanto al interior de los países como en la región en su conjunto, por medio de las campañas lideradas por las redes nacionales y regionales, lo que permitió potenciar las acciones e incidir exitosamente en el ámbito público.

Por otra parte, a partir de las reflexiones y acciones de las feministas en la región se produjo también un avance teórico y político importante, así como el desarrollo de metodologías y modelos de intervención integral, que consideran las jerarquías y los factores económicos, sociales, culturales, legales y psicológicos que están detrás de esta problemática. Se han realizado también acciones de seguimiento,

Relatoría Taller de la Red Feminista Latinoamericana y del Caribe contra la violencia doméstica y sexual. Propuestas para la Acción. Cancún, 8 de junio, 2001. 
auditoría y evaluación de las normas legales aprobadas y de las políticas ejecutadas, lo que ha permitido plantear modificaciones y mejoras a las acciones llevadas a cabo desde el Estado. La producción de conocimientos en la temática de la violencia sexista ha sido uno de los aportes más relevantes de las feministas, facilitando la reflexión y las acciones que permitieron posicionar esta problemática como una violación a los derechos humanos y como un asunto de justicia. Asimismo, se ha avanzado en visibilizar otras dimensiones de la violencia, como el hostigamiento sexual, la situación de las mujeres bajo conflictos armados y recientemente los asesinatos de mujeres o femicidios.

De esta forma, el movimiento feminista de la región se convirtió en un vehículo para la ampliación de la democracia y produjo algunas rupturas en el sistema de dominación patriarcal al permitir que las voces de las mujeres más excluidas (las abusadas y violentadas) pudieran ser un instrumento de inspiración y propuesta de reformas a los programas académicos, a las leyes y a las políticas estatales. Es decir, como lo han planteado algunas autoras, la problemática de la violencia contra las mujeres generó un discurso contra-hegemónico que diseminó nuevos conceptos e ideas en la esfera pública y promovió cambios y reformas en la agenda estatal (Marion, 2000; Weldon, 2004). En ese sentido, más que otros discursos propios del repertorio feminista, el discurso sobre la violencia contra las mujeres ha jugado un papel fundamental en los esfuerzos por "politizar" el ámbito de lo privado.

Lo anterior fue posible porque el Estado no es un ente monolítico e instrumental, sino un conjunto diferenciado de instituciones, un territorio en disputa que posee una autonomía relativa para la representación y reconstrucción de las relaciones de género (MacDowell, 2004; Schild, 1998). La relación entre el movimiento feminista y el Estado es dinámica, contradictoria y está determinada por la coyuntura política e histórica (MacDowell, 2004; Molineaux, 2000). Es decir, como cualquier movimiento social que intenta introducir cambios en la configuración del Estado y en la sociedad en su conjunto, el movimiento feminista tiene más posibilidades de incidir en los períodos y lugares más progresistas y de mayor apertura democrática. En ese sentido, las oportunidades para la incidencia feminista oscilan entre los momentos de cambio liberador y receptividad por un lado, $y$ los momentos de mayor conservadurismo $y$ represión, por otro (Lengermann y Brantley, 2000). Existe, además, una tensión permanente entre las propuestas $y$ visiones del movimiento feminista $y$ su capacidad de incidencia en los poderes establecidos. En otras palabras, en el terreno de las transformaciones sociales relativas a la violencia contra las mujeres, "no hay conquista definitiva ni avance sin contradicción" (Carcedo, 2003).

\section{LOS RETOS, LOS PELIGROS Y LAS PERSPECTIVAS FUTURAS}

La reconceptualización y posicionamiento de la violencia contra las mujeres como un asunto de justicia, ciudadanía y de derechos humanos por parte del movimiento feminista ha implicado importantes avances sociales. Sin embargo, como ya se planteó, hay una tensión permanente entre los planteamientos de cualquier movimiento social transformador y su incidencia en la agenda pública. En otras palabras, a medida que las propuestas del movimiento feminista ganan aceptación social, corren el riesgo de perder su carácter profundamente subversivo y transformador. De esta forma, las instituciones empiezan a apropiarse del discurso y planteamientos feministas, pero a acomodarlos a su lógica e intereses (CEFEMINA, 2003; Carcedo, 2003).

Por otra parte, la aprobación de las normas y leyes en América Latina no ha estado en consonancia con lo establecido en la Convención de Belém do Pará; es decir, no se ha aprobado legislación específica de violencia contra las mujeres, sino que se han aprobado leyes, normas y políticas relativas a la violencia doméstica o intrafamiliar, donde el bien protegido es la familia en abstracto $y$ no sus integrantes, como personas con cuerpos específicos ${ }^{5}$.

5 La excepción la representa Costa Rica al aprobar, en abril del 2007, la Ley de penalización de la violencia contra las mujeres adultas (Ley 13874). 
Además, el principal ámbito de acción de la nueva normativa es la prevención y la atención; es decir, no todas las leyes tienen un carácter sancionador, lo que implica la impunidad de la violencia y de quienes la ejercen. En general, la penalización de la violencia contra las mujeres se contempla o aplica solo cuando la conducta violenta es de cierta gravedad y constituye un delito tipificado en los códigos penales.

Asimismo, las normas desconocen la especificidad de la violencia contra las mujeres $y$ las relaciones de poder desiguales entre los géneros que se encuentran a la base del problema. Al ser planteadas como neutrales al género, esta normativa no sólo oscurece las particularidades de la violencia contra las mujeres, sino que, cada vez más, se revierten en contra de las propias afectadas al ser usadas por los agresores como un instrumento más de maltrato ${ }^{6} y$ por las instituciones como un mecanismo de revictimización (CEFEMINA, 2003). En ese sentido, a pesar de lo reciente de estas legislaciones $y$ políticas, su aplicación ya ha demostrado sus deficiencias y carencias.

La resistencia de los estados y de las instituciones sociales para abordar el problema específico de la violencia contra las mujeres se ve reflejada no sólo en la naturaleza de las leyes y políticas aprobadas, sino también en la forma en que operan las diferentes instancias encargadas de ofrecer servicios. La mayoría de las instituciones estatales tiene un enfoque básicamente asistencialista, desprovisto de una perspectiva de derechos $y$ de una visión articulada de proceso cuya meta sea la erradicación de la violencia. En estos servicios se trata a las mujeres como "beneficiarias" o "necesitadas de ayuda" o "víctimas" a las que hay que atender o soportar como un favor $y$ no como ciudadanas

Por ejemplo, se ha presentado el caso de agresores que, antes de que una mujer solicite medidas de protección contra ellos, según lo establece la Ley de Violencia Doméstica de Costa Rica, se adelan$\tan y$ presentan una denuncia contra la mujer, utilizando la misma ley. $\mathrm{O}$ de abusadores sexuales de niñas o niños que denuncian por violencia doméstica a su compañera y solicitan medidas de protección en su contra, por lo que ella es la que debe dejar la casa. con derechos, en particular a la protección, al debido proceso, a la integridad, a la justicia y a vivir libres de violencia.

La información recopilada en los distintos países apunta a que durante la tramitación de procesos, los funcionarios y operadores de justicia incurren con frecuencia en desempeños discriminatorios y sexistas, aplicando estereotipos basados en el género y funcionando bajo concepciones tradicionales, muchas veces, hasta forzando la conciliación entre las partes por encima de la protección de los derechos humanos de las mujeres. Lo anterior se constituye en un factor importante que causa severos problemas a las mujeres que intentan acceder a la justicia ya que la misma legislación de varios países ha privilegiado los mecanismos de conciliación (Brasil, Chile, El Salvador, Honduras, Perú, Ecuador y Venezuela), incluso fuera del ámbito judicial y sin considerar las relaciones desiguales de poder.

Lo anterior se convierte, en la práctica, en una forma de descriminilización de la violencia contra las mujeres y de desjudicialización de "asuntos" que no se consideran lo suficientemente importantes como para ser tratados directamente en el ámbito público de la justicia (Isis Internacional, 2002). Como lo plantean investigadoras feministas de Ecuador, que han visto las consecuencias de los procesos de conciliación:

Las salidas negociadas frente a la violencia debilitan la posición de las mujeres, quienes se ven forzadas a aceptar la impunidad como punto de partida $y$ posteriormente deben olvidar $y$ perdonar $y$, en ocasiones, incluso mantener el respeto por el agresor (Organización Panamericana de la Salud, 1997: 90).

Asimismo, en muchos de los países de la región, persiste una tendencia a minimizar los daños causados a las mujeres y a definir la conducta de los agresores como una falta menor, o a proponer el tratamiento psicológico como una pena alternativa.

Estas prácticas y concepciones institucionales ponen a las mujeres en una situación de gran desventaja frente a los proveedores de 
los servicios, lo que fomenta la impunidad y su revictimización por medio de la indiferencia, la trivialización, los cuestionamientos y hasta la burla de la que muchas veces son objeto (Sagot $y$ Carcedo, 2000).

Adicionalmente, las leyes y políticas no han reconocido la diversidad de mujeres y la multiplicidad de formas en que se manifiesta la violencia. En ese sentido, las manifestaciones específicas con que la violencia afecta a las mujeres de las diferentes clases sociales, edades, etnias, religiones, capacidades $\mathrm{u}$ orientaciones sexuales no se ven reflejadas en los instrumentos legales existentes. Por ejemplo, la normativa que se dirige a la niñez y la adolescencia no aborda la especificidad de género y existe una gran carencia de legislación y programas para prevenir $y$ atender la violencia sexual o el tráfico y la trata con fines de explotación sexual (Claramunt, 2003). A la vez, en muchos países de la región permanecen vigentes normas, como las que penalizan la interrupción voluntaria del embarazo, que representan formas abiertas de violencia contra las mujeres $y$ contra su derecho a la autonomía y la autodeterminación.

Respecto a la violencia sexual, se observa que en la región no se han tipificado sistemáticamente como delitos la violación conyugal o los ataques sexuales violentos dentro del hogar. En muchos casos, el ataque sexual sigue considerándose como un delito contra la moral y no como un delito violento que atenta contra la integridad personal de la víctima. Asimismo, en la mayoría de los ordenamientos jurídicos aún no se han tipificado como delitos los hechos de violencia sexual en contextos específicos, como es el caso del acoso sexual laboral, la violencia sexual en los espacios educativos, en los establecimientos de salud o en centros penitenciarios.

Por su parte, las políticas públicas relativas a la violencia ocupan un lugar marginal dentro de la política nacional. Estas políticas ni siquiera han sido incorporadas en los planes nacionales de desarrollo, ni se asumen como políticas de Estado; en ese sentido, tienen un carácter coyuntural y no representan compromisos nacionales de largo plazo. Por otra parte, estos planes tampoco cuentan con una adecuada asignación de recursos, ni con mecanismos de monitoreo y evaluación que permitan medir si de verdad los países avanzan hacia la meta de construir una sociedad que le garantice a las mujeres una vida libre de violencia. Desde esa perspectiva, la capacidad de atención, la cobertura y el número de servicios implementados, tanto desde la administración central como desde la local, son reducidos, no responden a la magnitud del problema o a las necesidades reales de las mujeres afectadas, $y$ muchas veces dependen de la cooperación internacional. Lo anterior se traduce en una relación de dependencia de los gobiernos, quienes aprovechan la temporalidad del financiamiento para no dar seguimiento a las políticas y programas, $y$ para no establecer compromisos de largo plazo.

Otra dificultad se relaciona con las limitaciones de financiamiento por las que atraviesan las organizaciones de mujeres, particularmente aquellas de los países en que se ha retirado gran parte de la cooperación internacional, como Costa Rica, y donde una difícil relación con el Estado hace aun más compleja la sustentabilidad de los programas existentes. Asimismo, en algunos países (El Salvador, Nicaragua y Honduras), los fondos que antes se dirigían a la atención de las mujeres ahora se están reasignando al tratamiento de agresores, con lo que se sacrifica la seguridad de las mujeres en función de un mecanismo que está lejos de demostrar su efectividad y que, más bien, tiende a fomentar la impunidad y a presentar a los agresores como víctimas de una violencia similar a la que sufren las mujeres.

La dinámica actual se da en un clima social mucho más conservador, caracterizado por el surgimiento de grupos organizados, tanto de carácter religioso como político, que intentan frenar los avances e incluso revertir los logros alcanzados por el movimiento feminista en la ampliación de la ciudadanía de las mujeres. Desde esa perspectiva, la situación actual de las organizaciones que luchan por enfrentar la violencia contra las mujeres puede ser leída como un símbolo de las nuevas condiciones en las que las feministas tratan de llevar adelante su agenda de transformación, pero se enfrentan a un Estado y a una sociedad menos receptiva, influenciada cada vez más por el discurso y la reacción organizada de grupos 
tradicionalistas que evocan el mantenimiento del orden de género (Sagot, 2006).

Desde la anterior perspectiva, es posible concluir que las relaciones del movimiento feminista con la sociedad en este terreno son problemáticas y están plagadas de contradicciones. Por un lado, se avanza en la incorporación de las demandas del movimiento en la agenda estatal y social, pero por otro, las propuestas feministas son vaciadas de su más importante aporte e instrumento de análisis: la concepción de la violencia contra las mujeres como un asunto de poder, producto de una sociedad estructurada sobre la base de la desigualdad y la opresión de género. De esta manera, los planteamientos radicales $y$ transformadores del movimiento feminista se "reciclan" en el Estado y en las instituciones, $y$ le son devueltos a la sociedad bajo la forma de leyes, políticas o programas socialmente aceptables y no amenazantes para el orden establecido. Muchas veces, entonces, en lugar de provocar transformaciones sociales y culturales, los procesos de institucionalización de las demandas feministas fomentan la cooptación de las propuestas y hasta del propio movimiento.

A pesar de lo anterior, la mayoría de las feministas y sus organizaciones creen que es importante seguir luchando por la aprobación de programas, leyes y políticas ya que son recursos que han abierto opciones para las mujeres maltratadas, ampliado sus derechos ciudadanos, salvado muchas vidas y contribuido a que se produzcan menos narraciones y representaciones falsificadas, parciales y perversas sobre la realidad social y las relaciones entre los sexos (Carcedo y Molina, 2003; CEFEMINA, 2003; Sagot, 2002). Es importante reconocer, además, que a pesar de los problemas y contradicciones, de los avances y retrocesos, los procesos de transformación social generalmente se van construyendo día a día y estos cambios promovidos desde el movimiento feminista ya empiezan a configurar una nueva concepción y práctica social sobre la violencia contra las mujeres, situación inimaginable hace 25 años.

Sin embargo, el objetivo del movimiento feminista no es $y$ no puede ser simplemente el luchar por mejores programas de atención, polí- ticas o leyes. El objetivo debe ser continuar con la lucha iniciada hace más de 200 años por la construcción de una sociedad que les garantice a las mujeres su autonomía, su bienestar y su integridad. Es decir, aún cuando de forma cotidiana se luche por la aprobación de una ley, de una política estatal o de mayores recursos para los programas de mujeres, nunca se debe perder el norte de la utopía que orienta al feminismo. Nunca se debe abandonar el instrumento analítico de las relaciones de poder, y sobre todo, en esta época de pragmatismo e individualismo exacerbado, nunca se debe olvidar la fuerza de las acciones colectivas progresistas y su efecto histórico en el destino de las sociedades. Esas acciones colectivas deberían dirigirse a hacer realidad la vieja aspiración feminista de construir una sociedad libre de todas las formas de violencia.

\section{BIBLIOGRAFÍA}

Carcedo, Ana y Karin Verbaken. La violencia contra las mujeres en Centroamérica y la Cooperación Holandesa. Costa Rica: CEFEMINA, 1992.

Carcedo, Ana y Alicia Zamora. Ruta crítica de las mujeres afectadas por la violencia intrafamiliar en Costa Rica. Costa Rica: Organización Panamericana de la Salud, 1999.

Carcedo, Ana y Montserrat Sagot. Femicidio en Costa Rica, 1990-1999. Costa Rica: INAMU-Organización Panamericana de la Salud, 2002.

Carcedo, Ana y Giselle Molina. Mujeres contra la violencia, una rebelión radical. Costa Rica: Embajada Real de los Países BajosCEFEMINA, 2003.

CEFEMina. Políticas públicas sobre violencia contra las mujeres: un balance desde las organizaciones de mujeres. Costa Rica: CEFEMINA, 2003. 
Claramunt, María Cecilia. Situación de los servicios médico-legales y de salud para víctimas de violencia sexual en Centroamérica. Costa Rica: Unidad Género y Salud, Organización Panamericana de la Salud, 2003.

Debert, Guita Grin. "As Delegacias de Defesa da Mulher: judicializaçao das relaçoes sociais ou politizaçao da justiça?”. Mariza Corrêa e Érica Renata de Souza (editoras). Vida em familia: uma perspectiva comparativa sobre "crimes de honra”. Brasil: Núcleo de Estudos de Gênero-UNICAMP, 2006.

Dobash, Rebecca E. y Russell P. Dobash. Violence Against Wives: a case against Patriarchy. New York: The Free Press, 1979.

. Women, violence and social change. London: Routledge, 1992.

Ellsberg, Mary C. et ál. Confites en el infierno. Prevalencia y características de la violencia conyugal hacia las mujeres en Nicaragua. Nicaragua: Departamento de Medicina Preventiva, UNAM-León, 1996.

Gordon, Linda. Heroes of their own lives: the politics and history of family violence. New York: Penguin Books, 1988.

Güezmes, Ana, N. Palomino y M. Ramos. Violencia sexual y física contra las mujeres en el Perú. Perú: Flora TristánOrganización Mundial de la SaludUniversidad Peruana Cayetano Heredia, 2002.

Heise, Lori, J. Pitanguy y A. Germain. Violencia contra la mujer: la carga oculta sobre la salud. Washington, DC: World Bank, 1994.

Isis Internacional. Violencia contra las mujeres en América Latina y el Caribe español
1990-2000: balance de una década. Santiago de Chile: Isis internacionalUNIFEM, 2002.

Johnson, Holly, N. Ollus and S. Nevala. Violence Against Women. An International Perspective. The United States: Springer, 2008.

Johns Hopkins University-CHANGE. Population reports: para acabar la violencia contra la mujer XXVII (4). Baltimore, MD: Johns Hopkins University, 1999.

Kennedy, Mirta. Violencia intrafamiliar. Ruta crítica de las mujeres afectadas en Honduras. Honduras: Organización Panamericana de la Salud, 1999.

Lazarus-Black, Mindie. The (heterosexual) regendering of a modern state: criminalizing and implementing domestic violence law in Trinidad. United States: American Bar Foundation, 2003.

Lengermann, Patricia M. y Jill N. Brantley. "Teoría feminista contemporánea". Teoría Sociológica Contemporánea. México: McGraw-Hill, 2003.

Lorentzen, Lois Ann y Jennifer Turpin. The Women and War Reader. New York: New York University Press, 1998.

MacDowell Santos, Cecília. "En-gendering the police: women's police stations and feminism in Sao Paulo". Latin American Research Review 39 (3). 2004.

Marion Young, Iris. La justicia y la política de la diferencia. España: Ediciones Cátedra, 2000.

Moller Okin, Susan. Justice, gender and the family. United States: Basic Books, 1989.

Molyneaux, Maxine. "Twentieth-Century state formations in Latin America”. Elizabeth 
Dore y Maxine Molineaux (editoras). Hidden Histories of Gender and the State in Latin America. Durham: Duke University Press, 2000.

Organización Panamericana de la Salud. Mujeres caminando, construyendo rutas para resolver la violencia intrafamiliar (Informe de Ecuador). Ecuador: CEPAMSENDAS-OPS, 1977.

Parsons, Talcott. Ensayos sobre teoría sociológica. México: Amorrortu, 1976.

Pleck, Elizabeth. Domestic tyranny: the making of social policy against family violence from colonial times to the present. New York: Oxford University Press, 1987.

Pola, M. J. Feminicido en República Dominicana. República Dominicana: Profamilia, 2002.

Rodríguez Saenz, Eugenia. Divorcio y violencia de pareja en Costa Rica (1800-1950). Costa Rica: EUNA, 2006.

Hijas, novias y esposas. Familia, matrimonio y violencia doméstica en el Valle Central de Costa Rica (1750-1850). Costa Rica: EUNA, 2000.

Sagot, Montserrat. "Marxismo, Interaccionismo Simbólico y la Opresión de la Mujer". Revista de Ciencias Sociales 63. Costa Rica, 1994.

Resultados de la Encuesta Nacional de Violencia Contra las Mujeres. Costa Rica: Centro de Investigación en Estudios de la Mujer, Universidad de Costa Rica, 2004.

. "La paz comienza en casa: las luchas de las mujeres contra la violencia y acción estatal en Costa Rica”. Natalie
Lebon y Elizabeth Maier (editoras). De lo privado a lo público. 30 años de lucha ciudadana de las mujeres en América Latina. México: Siglo XXI Editores-LASAUNIFEM, 2006.

Sagot, Montserrat y Ana Carcedo. Ruta crítica de las mujeres afectadas por la violencia intrafamiliar en América Latina. Costa Rica: Organización Panamericana de la Salud, 2000.

Schild, Verónica. "New subjects of rights? Women's movements and the contruction of citizentiship in the "new democracies". Sonia E. Alvarez, Evelina Dagnino y Arturo Escobar (editors). Cultures of politics/Politics of culture: revisioning Latin American social movements. Boulder, co: Westview Press, 1998.

Sullivan, Donna J. "Women's Human Rights and the 1993 World Conference on Human Rights". The American Journal of International Law 88 (1). 1994.

Tamayo, Giulia. Balance regional y desafíos sobre el derecho de las mujeres a una vida libre de violencia. Perú: CLADEMOXFAM, 2000.

United Nations. The world's women. Trends and statistics. New York: United Nations, 2000 .

Weldon, Laurel, S. "The dimensions and policy impact of feminist civil society". International Feminist Journal of Politics 6 (1). 2004.

World Health Organization. Multi-country study on women's health and domestic violence against women. Initial results on prevalence, health outcomes and women's responses. Switzerland: WHO, 2005. 